\title{
Moralische Skepsis, instrumentelle Vernunft und öffentliche Moral
}

\author{
Leist, Anton
}

DOI: https://doi.org/10.1515/9783110204605.97

Posted at the Zurich Open Repository and Archive, University of Zurich

ZORA URL: https://doi.org/10.5167/uzh-56173

Book Section

Published Version

Originally published at:

Leist, Anton (2003). Moralische Skepsis, instrumentelle Vernunft und öffentliche Moral. In: Leist, A. Moral als Vertrag? Beiträge zum moralischen Kontraktualismus. Berlin: De Gruyter, 97-120.

DOI: https://doi.org/10.1515/9783110204605.97 


\title{
Moralische Skepsis, instrumentelle Vernunft und öffentliche Moral*
}

\author{
Anton Leist
}

\section{Der unklare Ausgangspunkt}

In der Moralphilosophie ist die Frage, wie man das Begründen verstehen soll, die riskanteste Frage. Legt man sich nämlich auf ein bestimmtes Verständnis des Begründens fest, so ist es im weiteren kaum mehr zu korrigieren, legt allen Begründungsversuchen aber einen begrifflichen Rahmen auf. Andererseits muß man sich auf ein bestimmtes Verständnis des Begründens festlegen. Das heißt, man muß sich auch auf ein bestimmtes Verständnis der Moral festlegen. Einen nur formalen Begründungsbegriff gegenüber der Moral zu verwenden, ist nicht möglich, denn auch in diesem Fall würde man inhaltlich Stellung beziehen: nämlich das Begründen bei Wissensfragen im allgemeinen und das moralische Begründen gleichsetzen. Die Moral würde dann zu einer Art von Wissen.

Das Begründen der Moral ist auf zweierlei Weise mit einem bestimmten Verständnis der Moral verbunden. Erstens ist vor allem in einer aufgeklärten Moral ein Begründen selbst bereits Bestandteil der Moral, Alltagsmoral und Begründen zerfallen nicht in völlig getrennte Bereiche. Das vertiefte oder philosophische Begründen muß das alltägliche Begründen auf eine erhellende Weise erweitern, es darf ihm nicht völlig fremd sein oder es als solches sogar behindern. Zweitens sind sowohl das Begründen wie auch das Moralverständnis mit bestimmten Auffassungen des Zusammenlebens mit anderen Menschen verbunden. Diese Verbindungen sind leichter zu sehen bei der Alltagsmoral, schwerer beim Begründen. Ein Begründen setzt aber immer etwas voraus, und die verschiedenen Weisen des Begründens in der Moral wie der Moralphilosophie unterscheiden sich signifikant darin, was sie glauben, voraussetzen zu können.

Diese Hinweise darauf, daß jedes Begründen der Moral unausweichlich mit einem bestimmten Moralverständnis verbunden ist, könnten unter philosophisch Gebildeten so verstanden werden, als sollte damit ein Begründen im Sinn eines, Überlegungsgleichgewichts ${ }^{\mathbf{}}$ zwischen verschiedenen moralischen

* Für kritische Hinweise danke ich Holmer Steinfath, Peter Stemmer und Fritz Zimbrich. 
Ansichten vertreten werden. Ein solches Begründen ist im Alltag zwar üblich, es entspricht der Art und Weise, wie wir alltäglich in der Moral argumentieren und begründen. Aber es ist kein,vertieftes' Begründen. Ein vertieftes Begründen muß sich von den alltäglichen Ansichten stärker befreien, als es beim bloßen Streben nach Kohärenz möglich ist. Dazu muß es sich auf allgemeinste Verständnisweisen der letzten Elemente des Begründens und der Moral zurückziehen: auf ein - vereinfacht gesagt - Verständnis des Begründens unter sozial zusammenlebenden Personen.

Die verschiedenen Alternativen des Begründens der Moral werden manchmal als ,moralintern' und ,moralextern' unterschieden. Solche Unterscheidungen sind ähnlich mißverständlich und verwirrend wie Angaben zum Begründungsbegriff. Denn sie unterstellen ja bereits zu wissen, was Moral ist. Häufig nennt man eine Methode des kohärentistischen Begründens ,moralintern', eine rationale Begründung ,moralextern'. Aber es ist unklar, warum die Moral nur in moralischen Ansichten bestehen sollte, oder warum das Begründen oder die Vernunft nicht ebenso zur Moral hinzugehören sollten. Wenn es eine vernunftlose Moral je gegeben haben sollte - auf unser heutiges Moralverständnis trifft die Differenz jedenfalls nicht ersichtlich zu. Unverfänglicher ist es deshalb, von einem , alltäglichen` und einem ,vertieften` Begründen zu sprechen. Ob ein solches Begründen der Moral gegenüber extern sein kann oder muß, das ist erst zu ermitteln und nicht vorauszusetzen. Die klaren Unterscheidungen stehen am Ende der philosophischen Arbeit, nicht am Beginn.

Dieselben Bemerkungen gelten auch für die Beschaffenheit des ,moralischen Skeptikers', derjenigen Figur, die zu widerlegen oder zumindest in Schach zu halten, häufig als Kriterium des gelingenden Begründens angesehen wird. Für die Begründung ist es fatal, wenn von vornherein klar ist, was der Skeptiker fordert oder welche Art von Argumente er anerkennt und welche nicht. In solchen Fällen trägt der Skeptiker seinen Titel schnell zu unrecht, erweist sich nämlich als eine spezielle Sorte von Fundamentalist. Der ernst zu nehmende Skeptiker wäre hingegen derjenige, dessen Skepsis sich auch gegen seine eigene Frageposition richtet und der offen ist für eine Antwort, die seiner Skepsis nicht stärker ausgesetzt ist als seine Frageposition selbst.

Ist ein Begründen dann überhaupt möglich? Das ist vielleicht die verwirrendste dieser Vorfragen, die ebenfalls häufig mit dem Gegensatz von ,extern` ,intern' assoziiert wird: Es ist möglich, wenn vom externen Standpunkt aus, es ist unmöglich, wenn nur intern. Das ist schon deshalb ein irreführender Gegensatz, weil ja unklar bleibt, worüber geredet wird. ${ }^{1}$ Wiederum ist das

1 Zieht man die Analogie des empirischen Wissens heran, wäre der Gegensatz auch falsch: weil man nicht alles Wissen von außen begründen kann, ist es unbegründbar, und Wissen mit Wissen zu begründen, ist unmöglich? Natürlich lässt sich Wissen mit Wissen begründen, hingegen nicht Wissen mit Nichtwissen; und offensichtlich lassen sich (vergleichbar) spezielle Moralurteile mit allgemeineren begründen. 
weniger kategorische, eher graduelle Verständnis von vertieftem gegenüber dem alltäglichen Begründen hilfreicher. Ein vertiefendes Begründen stellt sich nicht in Gegensatz zur Moral, sondern erweitert unser bereits bestehendes Verständnis davon, was wir tun.

Eine klassische Variante dieses erweiterten Verständnisses ist die, das Begründen als eines gegenüber dem Egoisten aufzufassen. Der moralische Skeptiker ist danach ein Egoist. Eindeutig knüpft dieses Verständnis an den Alltag an, denn die Moral fordert uns häufig auf, unseren Egoismus zu überwinden. Allerdings zitieren wir im Alltag die Moral nur, um dem Egoisten $\mathrm{zu}$ antworten, wir begründen sie ihm gegenüber nicht. Wenn ein Egoist sagt, er wolle sein Versprechen nicht mehr halten, weil es für ihn nachteilig ist, so berufen wir uns ihm gegenüber auf die Moral, auf das zu haltende Versprechen, aber wir liefern ihm keine Begründung, warum man generell moralische Pflichten einhalten sollte. Im Alltag sind wir eher der Meinung, daß das nicht gelingen würde, wenn er die egoistische Haltung beibehält, und daß es vielmehr darum geht, ihn daran zu erinnern, daß er doch - im allgemeinen, irgendwie - moralisch ist.

In dieser Situation sind zwei Alternativen sichtbar. Einerseits, der lokale Egoismus wird zu einem globalen Egoismus verallgemeinert und $\mathrm{zu}$ dem (externen) Begründungsstandpunkt erhoben. Andererseits, das faktische Einbezogensein in die Moral wird in seinem Ausmaß und in seiner Bedeutung klarer vor Augen geführt und damit zu einem umfassenden (internen) Begründen verallgemeinert. Beides sind Methoden des vertiefenden Begründens, weil eine teilweise Erfahrung der Alltagsmoral systematisiert und die Alltagsmoral selbst entsprechend umorganisiert wird. Beide Methoden haben unterschiedliche strukturelle und inhaltliche Moralkonzeptionen zur Folge. Und beide Methoden sind allgemeinst-mögliche Methoden, schließen sich gegenseitig also aus.

Beiden Methoden gegenüber gilt je ein naheliegendes Bedenken. Erstens der Egoismus-Vorbehalt: angenommen, ein Begründen gegenüber dem Egoisten ist zwingend, inwiefern ist es relevant für uns, wenn wir nicht ebenfalls Egoisten sind? Denn wir sind kaum je vollständige Egoisten. Die beste Verteidigung des egoistischen Begründens ist vermutlich: Die Begründung reicht dann eben so weit, wie Du sie benötigst, insofern Du ein Egoist bist. In dieser Antwort steckt jedoch eine unbeantwortete offene Frage: wie wichtig sind die Grundmotive des Egoismus oder des Moralischseins als solche, und wie soll ich mich zu ihnen verhalten? Der Egoismus-Vorbehalt zielt eigentlich auf diese offene Frage.

Zweitens der Petitio-Vorbehalt: angenommen, unser Einbezogensein in die Moral kann verdeutlicht werden, inwiefern kann es dann zwingend sein? Unser Einbezogensein kann kontingent oder zwingend sein. Im ersten Fall benötigen wir weitere Gründe, um die Moral zu akzeptieren, die Begründung ist also nicht abgeschlossen. Im zweiten Fall scheint es sich nicht um eine 
übliche Begründung zu handeln, die offen läßt, daß man auch anders handeln kann. Daß wir notwendig in die Moral einbezogen sind, steht außerdem in Konflikt mit der Erfahrung des Egoismus und könnte bestenfalls in einer sehr allgemeinen, von der konkreten Moral abstrahierenden Weise gemeint sein. Doch wozu wäre ein solcher Nachweis dann gut?

Damit haben wir jedenfalls zwei Varianten des vertieften Begründens vor Augen. Der moralische Skeptiker kann sich als jemand erweisen, der nur durch egoistische Gründe zufriedengestellt wird, oder als jemand, der nur interne moralische Gründe zuläßt. Was beide Varianten des Begründens und ebenfalls beide Verständnisweisen der moralischen Skepsis teilen, ist die - wie es aussieht - pathologische Unbegrenztheit des Anspruchs. EgoismusVorbehalt und Petitio-Vorbehalt könnten auch mit einem Praxis-Vorbehalt zusammengefaßt werden: diese Versuche des Begründens müssen ins Leere laufen, weil sie das Begründungsproblem zu sehr verallgemeinern. Globale Begründungen in diesem Ausmaß sind weder sinnvoll noch erforderlich. Die Moral ist eine soziale Realität, und man muß sie nicht neu erfinden, sondern nur partiell verbessern. Dieser Vorbehalt steht dem Petitio-Vorbehalt offensichtlich näher als dem Egoismus-Vorbehalt.

In diesem Artikel wird es darum gehen, vor allem die externe oder egoistische Begründung kritisch zu beleuchten. Inwieweit der Petitio- und der Praxis-Vorbehalt berechtigt sind bzw. inwieweit das interne Begründen nur ein lokales Begründen sein kann, wird sich andeutungsweise ergeben. Eine völlig lokale Form des Begründens würde sich vom alltäglichen Begründen allerdings kaum unterscheiden und hätte dann keinen über den Alltag hinausgehenden Anspruch. Insofern geht es nur um die Wahl zwischen einem einzigartigen Begründungsstandpunkt und einer begrenzten Zahl von einander ergänzenden Begründungsgesichtspunkten. Das erste kann nur ,der' Standpunkt der praktischen Vernunft sein, letztere müssten wohl die einzelnen sozialen Güter sein, die unser soziales Leben wertvoll und verfolgenswert machen. Verschiedene Anforderungen an das philosophische Begründen erzwingen eine Synthese eines Vorrangs der einen praktischen Vernunft mit den verschiedenen sozialen Gütern, ohne daß im Augenblick genau gesagt werden könnte, wie das gelingen soll (s. auch Fn. 22).

\section{Moralische Skepsis und Egoismus}

Der moralische Skeptiker ist nicht bereits definitionsgemäß ein Egoist. Skepsis bedeutet auch Skepsis gegenüber den eigenen Voraussetzungen, einschließlich der begrifflichen. Warum sollte der Egoismus also ein Ausgangspunkt sein, um moralische Forderungen zu begründen? Eine Möglichkeit ist: aus empirischen Gründen, die Menschen sind überwiegend egoistisch, wenn es um soziale Beziehungen geht, und die Moral hat die soziale Funktion, diese Motive 
zu korrigieren. Eine andere Möglichkeit ist: aus begrifflichen Gründen, Moral und Egoismus schließen sich begrifflich aus, so daß die verallgemeinerte Skepsis vom Egoismus ausgehen muß.

Die erste Position muß man den empirischen (oder auch sozialwissenschaftlichen) Egoismus nennen, die zweite den prinzipiellen (oder begründungslogischen). Den empirischen Egoismus haben vor allem Hobbes und Hume vertreten, den prinzipiellen haben diverse Philosophen in der Absicht vertreten, einen von riskanten empirischen Annahmen freien, aber dennoch motivational gehaltvollen Ausgangspunkt zu gewinnen. ${ }^{2}$ Ein aktueller Vertreter des prinzipiellen Egoismus ist P. Stemmer (2000), auf dessen Position ich gleich zurückkomme.

Der empirische und der prinzipielle Egoismus unterscheiden sich in dem, was sie über durchschnittliche Menschen behaupten. Sie können deshalb unterschiedlich überprüft und kritisiert werden. Sie unterscheiden sich aber auch darin, wie sie das Begründen verstehen. Der empirische Egoismus versteht es als kollektives Begründen, der prinzipielle als individuelles. Ein kollektives Begründen ist eines, das Gründe für das Befolgen der Moral allen in einer Gemeinschaft gegenüber gibt, und soweit einzelnen gegenüber, so in Verbindung mit allen anderen einzelnen der Gemeinschaft. Hobbes und Hume hatten nicht vorrangig den ,Narren' oder den ,durchtriebenen Spitzbuben' im Sinn, obwohl auch im Blick. ${ }^{3}$ Beide sahen vorrangig auf das Kollektiv, für das es insgesamt besser ist, wenn Moralnormen befolgt werden als wenn nicht. Beide hatten gewisse Schwierigkeiten, den Einzelindividuen (Narr, Spitzbube) gegenüber eine zufriedenstellende Antwort zu geben.

Eine übliche Art, diese Einteilungen zu handhaben, ist die, sie entsprechend stehen zu lassen. Das kollektive Begründen nach Hobbes führt zur politischen, nicht zur Moraltheorie. Das kollektive Begründen nach Hume führt zur Sozialwissenschaft, nicht zur Ethik. Aber wie soll beides möglich sein, wenn die Moral jeweils ausgeschlossen ist? Die einzelnen Akteure müssten auch in diesen Theorien als Akteure verständlich werden, die sich gemäß der entsprechenden Politik oder der sozialen Realität rational verhalten können und diesen Nachweis haben sowohl die Hobbessche wie die Humesche Theorie nicht erbracht. Beide haben übersehen, daß auch empirische Diagnosen von der stimmigen Rationalisierung gegenüber allen einzelnen als einzelnen abhängen. Sieht eine empirische Theorie systematisch ein Rationalitätsdefizit

2 Diese Alternative ist, wie sich gleich zeigen wird, nicht so ausschließlich, wie es aussieht. Hobbes vor allem hat seine anthropologischen Urteile ja nicht auf Menschen im durchschnittlichen sozialen Leben, sondern im staatenfreien ,Naturzustand ' bezogen. Darin steckt ebenso eine hypothetische Bedingung, wie sich die begrifflichen Gründe auch auf reale Gesellschaften und Individuen beziehen müssen.

Lev. XV; EPM, IX, ii. 
bei den von ihr erfaßten Individuen vor, so ist sie, außer es handelt sich um geistig behinderte Menschen, letztlich auch empirisch nicht überzeugend.

Dieser Einwand läßt sich auch so formulieren. Daß sich die Menschen der Tendenz nach tatsächlich überwiegend egoistisch verhalten, kann kein binreichender Grund dafür sein, eine bestimmte Einstellung zur Moral anzunehmen oder nicht anzunehmen. Es kann ein Grund sein, wie man sich den anderen gegenüber verhält, wie man sich etwa vor ihnen schützt, aber es kann kein Grund dafür sein, ob man moralisch ist oder nicht. Zum Moralischsein gehört ein Verfügen über Gründe bezüglich des eigenen Handelns. Deshalb legt der verbreitete Egoismus ein bestimmtes Handeln nur nahe, wenn bereits ein Prinzip unterstellt wird, unter dem das Verhalten der anderen in Gründe des eigenen Handelns überführt wird. Dieses Prinzip, wie immer es inhaltlich beschaffen ist, folgt nicht selbst aus dem verbreiteten egoistischen Handeln. Menschen sind mit Gründen handelnde Wesen, und die tatsächliche Beschaffenheit ihrer Umwelt reicht nicht hin, um ihnen eine bestimmte Handlungsrationalität aufzuzwingen, im Unterschied zu Gründen, die sagen, wie wir gegenüber konkreten Anderen auf konkrete Weise handeln sollen.

Aus dieser Beobachtung ergibt sich auch das Verhältnis des empirischen Egoismus zum prinzipiellen. Die Beobachtung in anderer Formulierung: Für die Art der eigenen Gründe - im Unterschied zum Inhalt dieser Gründe - ist nicht wichtig, wie alle anderen sich verhalten. In ihrer Art bestimmen die Gründe, ob man selbst moralisch oder egoistisch sein soll. Der empirische Egoismus sagt bestenfalls etwas zum Inhalt, nicht zur Art von praktischen Gründen aus, und ist deshalb keine Begründungsposition, sondern nur eine mehr oder weniger komplexe Tendenzaussage. Wenn der Rest der Menschheit egoistisch wäre, wäre das kein guter Grund, selbst egoistisch zu sein - und nicht einmal, sich egoistisch zu verhalten. ${ }^{4}$ Demgegenüber zielt der prinzipielle Egoismus auf die Art der Gründe, oder darauf, wie man glaubt, daß idealerweise gehandelt werden sollte. Dabei kann er von empirischen Tendenzaussagen, zumindest auf einer grundsätzlichen ersten Ebene, absehen.

Wie muß man sich den prinzipiellen Egoisten vorstellen? Nach P. Stemmer führt der moralische Skeptizismus auf folgende Weise zum Egoismus. ${ }^{5}$ Die Alltagsmoral besteht in einer Menge von kategorischen Geboten, also von Geboten, die ungeachtet irgendwelcher vorauszusetzender Wünsche gelten. Der Skeptiker zweifelt an der Gültigkeit solcher kategorischen Gebote, denn er zweifelt daran, daß Gebote eine ähnlich objektive Existenz haben wie sonst

4 Man verhält sich egoistisch, wenn man egoistische Motive hat. Egoisten gegenüber muß man keine egoistischen Motive haben, es sei denn, man ist selbst ein Egoist. Man kann ihnen gegenüber so moralisch handeln, wie gegenüber allen, was natürlich nicht unbedingt darin besteht, die Wünsche der Egoisten zu erfüllen.

Vgl. Stemmer (2000) 17-19. 
empirische Dinge und Tatsachen. Damit beschränkt sich sein praktischer Standpunkt auf seine Wünsche, aus denen heraus er bereit ist zu handeln. Seine Wünsche lassen sich einteilen in die selbst- und in die fremdinteressierten Wünsche. Bei den ersten geht es um das eigene, bei den zweiten um das fremde Wohl. Die fremdinteressierten Wünsche, oder der Altruismus des Skeptikers, sind nicht stark genug, um das Wohl prinzipiell aller Menschen zu berücksichtigen. Die einzigen Wünsche, worauf sich eine Begründung stützen kann, sind deshalb seine selbstinteressierten Wünsche, oder sein Egoismus. Nur eine solche Begründung kann eine ,rational zwingende‘ Moralbegründung sein.

An dieser Darstellung könnte tendenziös oder sogar zirkulär erscheinen, daß ich den Standpunkt des Skeptikers, egoistisch' nenne. Ist ,Egoismus' nicht eine moralische Bezeichung, die in seinem Fall, da er sich von der Moral ja völlig distanziert hat, nicht mehr angewandt werden sollte? Aus solchen und ähnlichen Motiven heraus ist in der skeptischen Moraltradition auch von ,Selbstinteresse' oder von selbstinteressierten Wünschen die Rede. (Stemmer spricht außer von egoistischen auch von ,egozentrischen ${ }^{6}$ Interessen. $\left.{ }^{6}\right)$ Meines Erachtens handelt es sich bei diesem Punkt nicht nur um eine Frage der Terminologie, sondern auf versteckte Weise um die eigentliche Streitfrage: ob es nämlich einen Standpunkt im menschlichen Handeln gibt, der einerseits hinreichend ist, um von ihm aus das menschliche Handeln, einschließlich des moralischen, rational zu beurteilen und zu gestalten, und der andererseits klar noch nicht bereits ein moralischer ist. Die Proponenten eines „ja“ zu dieser Frage signalisieren ihre These mit der moralisch neutralen Terminologie; die Kritiker dieses „ja“ bzw. die Vertreter eines "nein“ beharren auf der Rede vom ,Egoismus', um die Unausweichlichkeit der Moral zu betonen. Ungeachtet dieses Unterschieds sollte die moralisch neutrale Rede aber zunächst zugestanden werden.

Stemmer redet sowohl von ,egoistischen' wie ,altruistischen' Wünschen, und in dieser Gegenüberstellung muss „egoistisch“ in seinem Sinn wertneutral verstanden werden. Egoistische Wünsche sind dann einfach nur solche, in denen das eigene Wohl der Gegenstand ist, altruistische solche, in denen das Wohl anderer der Gegenstand ist. Dieser Verwendung schließe ich mich hier ebenfalls an.

Eine weitere, hier anschließende Frage betrifft das Gewicht, das die egoistischen bzw. altruistischen Wünsche haben sollen. Stemmer ist der Meinung, daß einzig die egoistischen Wünsche als Begründungsstandpunkt in Frage kommen, nicht (auch) die altruistischen. Die altruistischen Wünsche seien 
nicht zwingend, die egoistischen schon. Da wir rein faktisch sowohl die einen wie die anderen Wünsche haben, ist das eine schwierige Wahl. Ich möchte auf sie erst zurückkommen, wenn etwas mehr Klarheit über die Relevanz der Wünsche im allgemeinen geschaffen ist.

\section{Die Fiktion der rationalen Wunschtheorie}

Das eigentliche Problem des soweit geschilderten moralischen Skeptikers besteht nämlich nicht darin, wie die bei ihm noch vorhandenen Wünsche genauer beschrieben werden sollen, sondern darin, daß ihm zuviel abhanden gekommen ist, wenn er sich nur noch auf seine Wünsche stützen kann. Wenn wir von Wünschen reden, meinen wir tatsächliche Begehrzustände, die nicht nur aktuell, sondern auch dispositionell sein können, die aber als solche nicht mehr sind als psychische Fakten. Wie andere Fakten auch sind psychische Fakten per se noch keine Gründe. Die vorhin beachteten Verhältnisse wiederholen sich hier: Wünsche können Prämissen innerhalb von Gründen sein, aber um $\mathrm{zu}$ vollständigen Gründen zu werden, müssen andere, sich nicht nur auf Tatsachen beziehende Prämissen hinzukommen. Die Art und Weise, wie in der Humeschen Tradition der moralische Skeptiker geschildert wird, läßt aber keinen Spielraum, um solche zusätzlichen Prämissen einzuführen.

Damit soll nicht behauptet werden, daß sich die typischen Humeaner (wie Stemmer) nur auf Wünsche stützen. Der Standpunkt des moralischen Skeptikers muß ja irgendwie auch als rationaler Standpunkt ausgewiesen werden, um ihm die angemessene normative Verbindlichkeit zu geben. Es muß gesagt werden können, warum der außermoralische Standpunkt ein praktisch vernünftiger ist bzw. warum man von ihm aus Gründe hat, moralisch zu sein. ${ }^{7}$ Stemmer befindet sich in Übereinstimmung mit den häufig erwähnten Forderungen an ,rationale Wünsche', wonach solche Wünsche dann vorliegen, wenn sie innerhalb der eigenen Wunschmenge kohärent sind und außerdem mit den eigenen Meinungen und verfügbaren Informationen harmonieren. ${ }^{8}$ Rationale Wünsche hat man demnach dann, wenn die Wünsche, die man hat,

7 Im folgenden werden „rational“ und „vernünftig“ bzw. „Rationalität“ und „Vernunft“ immer gleichbedeutend verwendet.

8 Stemmer (2000) 25-29. Der erste, der - anschließend an Humes kursorische Bemerkungen - dieses empirisch-kognitiv rationale Verständnis rationaler Wünsche genauer formuliert hat, war Brandt (1979) bes. Kap. 6-8. Seither wird das empirisch-kognitive Rationalitätsverständnis von Wünschen innerhalb der, überlegten-Wünschetheorien' nur noch in Details variiert. Ein strittiger Punkt ist etwa, ob die Informationen subjektive oder objektive sein sollen. S. zu einer inspirierenden, wenn auch unübersichtlichen Diskussion rationaler Wünsche Parfit (1984) Kap. 6. 
nicht ,kognitiv defizient' sind, wenn sie den übrigen Wünschen, Meinungen und Informationen nicht widersprechen. Es scheint, daß auf diese Weise alle Wünsche und Interessen rational sein können.

Manche Menschen haben eigenartige Wünsche wie das Essen einer Handvoll Schlamm oder das Fahren in einem sehr schnellen Auto. Nach dieser kognitiven Wunschtheorie würden solche Wünsche, wäre ihre Ausführung nur entsprechend riskant, mit dem Wunsch nach einem guten Leben kollidieren und damit als irrational erklärt. Viele Menschen haben mehr oder weniger klare und starke Wünsche nach einem guten Leben. Stemmer macht explizit deutlich, daß die empirisch-kognitive Konzeption von Wünschen ,wertfrei ${ }^{6}$ ist, also keine in sich wünschenswerten Sachverhalte unterstellt. Und er betont außerdem, damit verbunden, daß die Rationalität der Wünsche nur in dem Wie des Zustandekommens der Wünsche liegen kann, nicht in einer inhärenten Qualität, einem Was der Wünsche. ${ }^{9}$ Diese Behauptung ist allerdings nicht ganz richtig, denn wie eben gesehen, wird ja dennoch ein ,Was' der Wünsche unterstellt - eben in Form derjenigen Wünsche und Interessen, von denen angenommen wird, daß alle Menschen sie haben, wie etwa das Lebensinteresse oder ein Interesse an einem guten Leben.

Inwiefern kann dieser Standpunkt für sich in Anspruch nehmen, rational zu sein, wenn er von Interessen abhängt, die man einfach nur hat? ${ }^{10}$ Damit sind wir bei dem Problem der Interessen als grundlegender Fakten. Nach der rationalen Wunschtheorie sind diese Interessen nicht nur tatsächlich gegebene Interessen, sondern auch, rationale Interessen', weil (und wenn) sie nicht kognitiv-defizient sind. Das faktische Gegebensein und der rationale Anspruch sollen auf diese Weise miteinander vereinbart werden. Allerdings bestenfalls in einem formalen Sinn. Es leuchtet ein, daß gegenüber dem Wunsch, eine Handvoll Schlamm zu essen, eine Art ,rationaler Kontrolle“ ausgeübt wird, wenn Folgen dieses Essens und das Lebensinteresse gegen den Wunsch sprechen. Welche Art von Kontrolle wird aber gegenüber dem Lebensinteresse selbst ausgeübt, wenn man sagt, es sei nicht ,kognitiv-defizient'? Soweit ich sehe, keine - das Lebensinteresse wird einfach als arationales Faktum unterstellt, und es wird nicht rationaler dadurch, daß man es mit sich selbst vergleicht. In der Folge übertrüge aber das Faktum des Lebensinteresses seine Eigenschaft, arational zu sein, auf alle speziellen Wünsche, die mit seiner Hilfe kontrolliert werden.

Die rationale Wunschtheorie könnte mit dem Hinweis verteidigt werden, daß eben dieses eine Faktum vorausgesetzt werden müsse, ansonsten aber

9 Stemmer (2000) 29-30.

10 Häufig wird in diesem Zusammenhang auch gesagt: „die viele oder nahezu alle Menschen haben“. Damit fiele der prinzipielle Egoismus aber auf den empirischen zurück. 
zurecht ein rationaler Anspruch bestehe. ${ }^{11}$ Diese Verteidigung mißlingt deshalb, weil erstens mindestens das Interesse an einem guten Leben unterstellt werden müsste, und nicht einfach ein Interesse nach Leben, und zweitens dieses Interesse nicht klar abgegrenzt oder benannt werden kann, was heißt, $\mathrm{da} ß$ es genaugenommen mit einer offenen Menge von Wünschen identisch ist. Der erste Punkt ist leicht zu sehen: Der Wunsch, mit einem sehr schnellen Auto zu fahren, mag im Licht des Lebensinteresses kognitiv-defizient sein, aber der Wunsch, das ganze Wochenende ziellos mit dem Auto durch die Stadt zu fahren, ist es keineswegs. Dennoch erscheint uns dieser Wunsch rational zweifelhaft, wie viele andere Wünsche sinnlosen Tuns, auch wenn sie nicht unmittelbar gefährlich oder lebenszerstörerisch sind. Menschen unterscheiden sich von Tieren eben gerade darin, daß sie auch in elementarster Betrachtung nicht einfach nur leben wollen, sondern qualifiziert leben wollen. Ein bloßes, qualitätsloses Leben gibt es für Menschen, jedenfalls unter normalen Umständen, nicht.

Wird dieser erste Punkt zugestanden, ist die rationale Wunschtheorie nicht mehr zu retten. Menschen unterscheiden sich von Tieren dadurch, daß sie sich ihre Ziele geben. Das wäre unmöglich, einzig unter Rekurs auf ein blankes Lebensinteresse. (Es wäre auch unmöglich, unter Rekurs auf eine beliebige Zahl sonstiger ,blanker', also selbst qualitativ unbestimmter Interessen.) Es wäre natürlich auch unmöglich, durch Rekurs auf eine fixe Menge irgendwelcher Werte. Vielmehr stellt sich Menschen ihr Lebensinteresse als qualifiziertes Interesse immer im Kontext von Entscheidungen über qualifizierte Wünsche dar. Es scheint zwar so, daß wir jenseits der verschiedenen Lebensmöglichkeiten immer voraussetzen, daß wir nur irgendwie leben wollen, das bloße Lebenwollen also das Wichtigste wäre. Aber wie man in Extremsituationen und im Alter erfährt, stimmt das nicht. Und selbst wenn es stimmen würde, wäre es doch keine Garantie für die rationale Qualität des entsprechenden Lebens. ${ }^{12}$

Die empirisch-kognitive Wunschtheorie kann also überhaupt nicht sagen, was praktische Vernunft sein soll. Sie kann bestenfalls anhand lokaler Fragen den Schein erzeugen, rationale Wünsche ließen sich von irrationalen unterscheiden. Der Standpunkt der Moralbegründung ist jedoch keiner, der mit einem alltäglichen, lokalen Problem zu tun hätte. Die Annahme ist deshalb illusionär, der Skeptiker könnte als jemand beschrieben werden, der seine

11 Und muß nicht angenommen werden, daß sich rationale Gründe auch auf tatsächliche Zustände beziehen oder solche Zustände sind, denn sie gelten ja für tatsächliche Menschen?

12 Jemand lebt trotz seiner Schande weiter, obwohl er wünschte, er täte es nicht. Oder jemand lebt trotz seiner Qualen weiter, obwohl er den Tod wünscht. Wären das hinreichende Gründe, das entsprechende Leben rational zu nennen? 
Wünsche empirisch-kognitiv prüft, um dann zu dem Ergebnis zu gelangen, er habe eine bestimmte Menge egoistischer und altruistischer Wünsche. Gegenüber so umfassenden Orientierungsfragen funktioniert die empirischkognitive Prüfung nicht, weshalb in der Beschreibung des außermoralischen skeptischen Standpunkts zwei unvereinbare Teile - empirische Tendenzaussagen zur Motivation und die empirisch-kognitive Wunschtheorie - miteinander vermischt werden. Genaugenommen kann ein Humeaner (wie Stemmer) überhaupt nicht sagen, welche Wünsche der moralische Skeptiker als rationale Wünsche hätte. Er verfügt über keine Mittel, dazu eine Aussage zu machen.

\section{Gründe statt Wünsche}

Wie sollte man sich den moralischen Skeptiker also besser vorstellen? Der Hinweis darauf, wie man sich den Skeptiker eigentlich vorstellen muß, ist enthalten in der Alternative des Was der Wünsche, im Unterschied zum Wie. Für die empirisch-kognitive Konzeption scheidet diese Alternative deshalb als erwägenswert aus, weil man befürchtet, in eine objektivistische Wertauffassung zurückzufallen, von der sich der Skeptiker gerade befreien will. Dieses Bedenken ist verständlich, allerdings nicht ausreichend, um die inhaltlichrationale Qualität von Wünschen völlig zu verwerfen. Die Humeaner sind von einem Zerrbild der Wertontologie gefangen, nach der die einzige Alternative zum (naturalistischen) Wert anhand tatsächlicher Wünsche in der objektiven Existenz intrinsischer Werte liegen soll. Wenn Wünsche nicht über ihre Entstehungsbedingungen, sondern über ihre Ziele und Gegenstände wertvoll sein sollen, so nach dieser Vorstellung nur deshalb, weil die Ziele und Gegenstände selbst , an sich` wertvoll sein müssen. ,An sich' wertvolle Gegenstände, in der Welt ${ }^{\star}$ erscheinen den Humeanern mysteriös und sind deshalb abzulehnen. ${ }^{13}$ Übersehen wird dabei, daß die Werthaftigkeit der Ziele dadurch zustandekommen kann, daß sie im Vergleich mit anderen Zielen in einem Raum von Gründen stehen. ${ }^{14}$

13 Mackie war bekanntlich der Meinung, daß die Eigenartigkeit („queerness“) der objektiven Werte durch wissenschaftliche Konkurrenz mit der rationalen Wunschtheorie erwiesen werden sollte: Mackie (1977) Kap. 1. Gegeben die Schwierigkeiten der rationalen Wunschtheorie, wären nach diesem Kriterium die vermeintlich rationalen Wünsche nicht weniger „queer" als die vermeintlich objektiven Werte. Aber Mackies Metakriterium beruht sowieso auf einer Überbewertung der Wissenschaften. Ontologische Fragen können nicht empirisch entschieden werden.

14 Die nachfolgenden Bemerkungen sind inspiriert von Scanlon (1998) Kap. 1. Hinweise auf praktische Gründe als Ziel- oder Gegenstandsaspekte von Wünschen finden sich auch bei Parfit (1984) 123: „It is irrational to desire something that is in no respect worth desiring, or is worth avoiding." (Parfit scheint allerdings die allgemeine Bedeutung der (wie er sie nennt) 
Die Alternative ist die: Wünsche sind nicht verfolgenswerte oder rationale Wünsche, weil sie mit anderen Wünschen und Informationen kohärieren, sondern weil mit ihnen ein Grund verbunden ist, der nicht global dem Wunsch, sondern dem Wunschgegenstand entspringt. Der Wunsch, mit einem schnellen Auto zu fahren, ist dann ein rationaler Wunsch, wenn schnelles Autofahren ein Grund ist, diesen Wunsch zu realisieren. Ein nicht nur rationaler, also durch Gründe getragener Wunsch, sondern ein guter und verfolgenswerter Wunsch liegt dann vor, wenn Gründe und Gegengründe insgesamt dafür sprechen, den Wunsch zu realisieren. Wann sprechen diese Gründe dafür oder dagegen, im konkreten Fall des schnellen Autofahrens? Ist dieses Autofahren per se wertvoll, so daß sich alle Gründe nach diesem Wert richten müssen? Das ist natürlich der potentielle Verdacht der Humeaner.

Der Verdacht ist unbegründet: indem wir etwa das Rauschgefühl des schnellen Autofahrens als Grund nennen, erheben wir es zu einem Wert, der rechtfertigt, es in die Tat umzusetzen. Wie gelingt uns das? Es gelingt uns einmal durch Bezugnahme auf die Erfahrung selbst, die Behauptung und den Genuss des Gefühls, und zum andern durch Vergleich mit anderen Erfahrungen. Welchen Wert etwas hat, oder ein wie guter Grund es ist, etwas zu wünschen und zu tun, lässt sich nur im Vergleich mit anderen Erfahrungen feststellen, die alternativ möglich wären. Mit Kindern spielen könnte etwa besser sein, wie diejenigen beurteilen können, die beides beherrschen, und das geringere Risiko und die Vor- bzw. Nachteile für andere (Kinder, Verkehrsteilnehmer) könnten ein definitives Übergewicht der Gründe herbeiführen. Selbst wer von dieser Werttheorie, der Auflösung von Werte in Gründe, metaethisch nicht überzeugt ist, sollte deshalb zugestehen, daß praktische Entscheidungen nur anhand der Gegenstände des Wünschens möglich sind, und nicht über einen Test der Kohärenz von zugrundliegenden Wünschen. ${ }^{15}$

Zumindest skizzenhaft haben wir damit zwei alternative Rationalitätsauffassungen vor Augen. Soweit ein rationales Verhältnis zur Moral betroffen ist, ergibt sich aus ihnen die Alternative, die Moral entweder unter dem Gesichtspunkt der vorauszusetzenden Interessen oder unter dem ,Gegenstand' der Moral, den sozialen Beziehungen, zu betrachten. Für die weitere Diskussion scheint das die erhellendere Alternative als die beliebte Frage: „wieviel von der Alltagsmoral kann der Kontraktualismus retten?" Vor allem dann, wenn

„critical present-aim theory“ im Rahmen der von ihm kritisierten Selbstinteresse-Theorie verborgen geblieben zu sein.) Scanlon (1998, 378, Fn. 27) verweist auf eine Reihe von weiteren Autoren.

15 Könnte die rationale Wunschtheorie nicht Information zu den Wunschgegenständen in den kognitiven Defizienztest einbeziehen? Nur in ihrer Funktion, Wünsche zu korrigieren, nicht in der Funktion, Wünsche allererst zu erzeugen. 
man die letztere Frage nur quantitativ versteht, etwa das Ausmaß auf diese Weise erreichbarer moralischer Gleichheit zu ermitteln sucht, muß die Debatte im Nirgendwo versanden. Die Kontraktualisten können damit leben, daß sie eine relativ begrenzte Moral anbieten, ${ }^{16}$ und die Diskussion über die Grenze eines moralischen ,Minimums' ist kaum entscheidbar, weil keine Partei über eine begründungsunabhängige Definition für Moral verfügt. Demgegenüber dürfte sich leichter zeigen lassen, daß der Kontraktualismus Schwierigkeiten hat, überhaupt ein angemessenes Verständnis von sozialen Beziehungen zu finden.

\section{Vernunftbegriff und soziale Beziehungen}

Die entsprechende Schwäche des instrumentellen Vernunftmodells haben wir nämlich bereits ausgemacht und es gilt eigentlich nur, daraus die weiteren Konsequenzen zu ziehen. Denn: Indem bestimmte Handlungen, Motive oder Wünsche als, rational' bezeichnet werden, müssen bestimmte Interessen vorausgesetzt werden, von denen nur gesagt werden kann, daß sie gegeben, nicht daß sie selbst ebenfalls ,rational' sind. Dieses Modell ist den menschlichen Lebensverhältnissen nicht angemessen, auch nicht den sozialen Beziehungen. Die Diskussion auf die Ebene der sozialen Beziehungen zu verlagern, hat den Vorteil, daß wir schneller zu sehen beginnen, was das instrumentelle Modell bzw. der Kontraktualismus in der Moral praktisch bedeuten. Im folgenden nenne ich den ,Instrumentalisten ' denjenigen, der nur den instrumentellen Vernunftbegriff zur Verfügung hat. Es geht darum, zu sehen, was das sozial bedeutet.

Den soweit vorgebrachten Einwand, daß die grundlegenden Interessen nicht selbst rational sein können, kontert der Instrumentalist üblicherweise damit, daß das prinzipiell unvermeidbar ist. Die menschliche Natur ist in einem unausweichlichen Sinn vorgegeben und praktische Gründe müssen an diese Natur anknüpfen. In einem bestimmten Ausmaß können die Menschen ihre Interessen nicht erfinden, sondern sie haben sie unveränderlich. Damit hat der Instrumentalist zweifellos recht, er zieht allerdings eine falsche Folgerung. Denn der Verweis auf die vorgegebene menschliche Natur muß nicht so verstanden werden, daß die menschlichen Interessen und Motive gleichsam aus zwei inhaltlich trennbaren Schichten bestehen, deren eine gegeben und deren andere instrumentell regulierbar wäre. Alles, was wir mit Handlungsbegriffen wie ,Interesse‘ usw. bezeichnen, ist jeweils sowohl gegeben wie auch veränderbar. Der Instrumentalismus scheitert an dieser Doppelaspektualität

16 S. etwa Stemmer (2000) $\mathbb{S} 8$ 8-9. 
der menschlichen Eigenschaften, weil er eine inhaltliche Trennbarkeit voraussetzt.

Der Instrumentalismus und die Trennung von Gegebenem und Normativem sind eindeutig Bestandteil der kontraktualistischen Moral. Die Interessen, egoistische und altruistische, sind gegeben und die moralischen Normen sollen auf dieser Basis begründet und in bestimmter Hinsicht gestaltet werden.

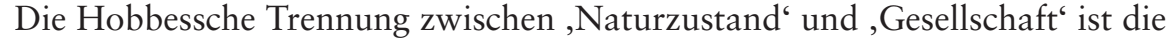
extremste Veranschaulichung dieses inhaltlichen Trennungsbemühens, hypothetische Kontraktualisten wie Stemmer müssen die Trennbarkeit aber zumindest implizit ebenfalls unterstellen. Analog können wir uns klarzumachen versuchen, was der Instrumentalismus für die sozialen Beziehungen bedeuten würde. Die hypothetischen Kontraktualisten sprechen zwar nicht offiziell von sozialen Beziehungen, aber warum sollten wir ihre hypothetischen Gründe als für uns relevant ansehen, wenn ihnen jeder tatsächliche soziale Gehalt abginge? Können wir den instrumentell gedeuteten sozialen Beziehungen in keiner Weise zustimmen, spricht auch nichts dafür, hypothetisch Instrumentalist zu sein.

Eine andere Verteidigung des Instrumentalisten lautet aber so. Der Instrumentalist versteht sich als jemand, der nur einen instrumentellen (humeanischen) Vernunftbegriff zur Verfügung hat. Dieser Begriff ist wesentlich ein Begründungsbegriff, der verständlich machen soll, warum Handlungen rational sind. Darin ist er so allgemein, daß er neutral ist gegenüber bestimmten Formen des sozialen oder nicht-sozialen Handelns. Er ist mit verschiedensten inhaltlichen Formen dieses Handelns vereinbar. Deshalb ist es auch berechtigt, daß die Ethik von den Formen sozialen Handelns abstrahiert und nicht auf einer konkreten sozialen Handlungstheorie aufbaut. Beobachtungen zu den sozialen Beziehungen sind deshalb für eine kontroverse Diskussion über den Rationalitätsbegriff oder die Moral irrelevant. Die Ethik kommt zurecht ohne sie aus.

Die Verwendung des Begriffs „instrumentell“ kann sicher leicht dazu verführen, unberechtigte Analogien herzustellen. Ein ,um-zu-Verhältnis‘ bei Motiv und Handlung und bei sozialen Beziehungen kann jeweils eine andere Qualität besitzen. Im einen Fall kann es elementar und unausweichlich sein, im anderen tendenziös, weil nicht unausweichlich. Wenn ich $\mathrm{x}$ tue, um den Wunsch y zu befriedigen, so kann das unausweichlich sein, weil ohne einen Wunsch nicht gehandelt werden kann. Wenn ich eine Person B benutze, um x zu erreichen, so kann das problematisch sein, weil B zu mehr imstande ist, als nur benutzt zu werden, oder weil das Benutzen die Handlungsmöglichkeiten gegenüber B auf eine Weise einschränkt. Der instrumentelle (wenn man ihn so nennen will) Rationalitätsbegriff $m u ß$ also nicht zu einem instrumentellen Verständnis sozialer Beziehungen führen.

Ich denke, daß diese Beobachtung richtig ist. Das Defizit des instrumentellen Rationalitätsbegriffs liegt nicht darin, daß er notwendig zu instrumen- 
tellen sozialen Beziehungen führt. Es liegt vielmehr darin, daß er die Qualität von sozialen Beziehungen nicht erhellen kann und daß er, damit verbunden, gegenüber instrumentellen sozialen Beziehungen neutral bleibt. Das Defizit des instrumentellen Begriffs liegt darin, inhaltliche Interessen als gegebene unveränderlich vorauszusetzen, und damit in seiner Rationalisierungsbegrenzung. Gegenüber den vorauszusetzenden Interessen kann nicht gesagt werden, daß sie rational sind oder auch nicht. Darin ist der instrumentelle Begriff tatsächlich neutral gegenüber den tatsächlichen Interessen, aber das ist eine Schwäche des Begriffs.

Betrachten wir nämlich nur den Gegensatz von egoistischen und altruistischen sozialen Beziehungen. Egoistische Beziehungen, nehmen wir an, sind solche, in denen mit dem anderen gehandelt wird, um ein Ziel zu realisieren, das einem selbst nützt. Daß es anderen auch nützt, muß nicht ausgeschlossen werden, ist aber nicht Bestandteil des Handlungsmotivs. Altruistische Beziehungen sind solche, in denen gehandelt wird, um ein Ziel zu realisieren, das anderen nützt. Daß es einem selbst nützt, muß nicht ausgeschlossen werden, ist aber nicht Bestandteil des Handlungsmotivs. Der instrumentelle Begriff kann zwischen diesen Alternativen nicht unterscheiden und jemand, der nur egoistische oder nur altruistische Beziehungen hätte, kann nach ihm nicht unvernünftig genannt werden. Diese Begrenzung ist deshalb nicht belanglos, weil es wichtiger ist, daß Rationalität uns sagt, wie wir uns insgesamt verhalten sollen, sogar wie wir insgesamt leben sollen, als wie wir in einzelnen, je speziellen Situationen zu handeln haben.

Den instrumentellen Vernunftbegriff , neutral' ${ }^{`} \mathrm{zu}$ nennen, ist sogar leicht beschönigend. Genaugenommen ist er , beratungs-opportunistisch'. Als Egoist handle ich aus den egoistischen Interessen und das ist, rational', sofern ich eben diese Interessen habe. Als Altruist ebenso, wenn ich eben altruistische Interessen besitze. Als beliebige Person in einer beliebigen Rolle handle ich rational, wenn ich mir einfach die entsprechenden Interessen unterstellen kann. Das wäre dann kein eigentliches Defizit, wenn bestimmte Interessen in Menschen unveränderlich und unwählbar eingepflanzt wären und deshalb keiner Beratung bedürften. Für Menschen ist aber typisch, daß selbst die stärksten Interessen, wie der Lebenswunsch oder die Furcht vor Schmerzen und sozialer Einsamkeit, in manchen Situationen der Beratung bedürfen. Umso mehr alle übrigen Wünsche, die keineswegs aus den stabileren Interessen einfach abgeleitet werden können. ${ }^{17}$ Der instrumentelle Begriff läßt den

17 Die spezielleren Interessen erweitern die allgemeinen und sind in ihnen nicht bereits enthalten. Daß ein deduktives Modell für die Beziehung zwischen allgemeinen und speziellen Interessen unangebracht ist, spricht wiederum gegen die Schichten-Trennung, wie sie mit dem instrumentellen Begriff einhergeht. 
größten Teil unseres Handelns nicht nur arational, sondern beliebig erscheinen. Und das ist vor allem ein empfindliches praktisches Defizit.

Das größte Defizit der kontraktualistischen Moralbegründung besteht deshalb auch darin, daß sie nichts dazu zu sagen hat, inwieweit wir eher Egoisten oder eher Altruisten sein sollten. Da man nicht einfach annehmen kann, wir seien in einem konkreten Ausmaß bereits je das eine oder andere, ist das eine erhebliche Schwäche. Obwohl sich Stemmer auch in seinen Bemerkungen zum Altruismus weitgehend am instrumentellen Rationalitätsbegriff orientiert, ${ }^{18}$ ist er dennoch bemüht, den Bereich der altruistischen Gefühle und Ideale zu erweitern, also dafür zu plädieren. Ein solches Bemühen ist jedoch unverständlich und inkonsequent. Man kann es nicht aus den tatsächlichen altruistischen Motiven heraus begründen, weil diese Motive keine reflexive Dimension umfassen sollen, aus denen heraus sich sagen ließe, es sei gut, sie zu haben. Der Altruist handelt unmittelbar aus altruistischen Motiven, ohne daß er sagen könnte, es sei gut, solche Motive zu haben. Wie es ist, im Sinn eines Werturteils, egoistische oder altruistische Motive zu haben, darüber muß der Instrumentalist schweigen. Er hat diese Motive de facto, oder er hat sie nicht; er kann nur darum bemüht sein, ihnen entsprechend möglichst effektiv $\mathrm{zu}$ handeln. In der realen Welt ist die instrumentelle Rationalität deshalb als beratende irrelevant. Sie sagt uns nichts zum Selbstverhältnis gegenüber unseren schwankenden und beratungsbedürftigen Interessen und Motiven. ${ }^{19}$

Der Instrumentalist könnte natürlich darin recht haben, daß es keine alternative Form der Rationalität gibt. Er könnte diese ganze Kritik zugestehen und nur die Hoffnung äußern, daß die menschlichen Interessen und Motive de facto stabiler sind, als sie hier geschildert werden. Tatsächlich sind sie bei vielen Menschen im Alltag stabiler als es im philosophischen Begründungsdiskurs scheinen könnte. Daß der instrumentalistische Vernunftbegriff diese Stabilität nicht erklären kann, sondern einfach unerklärt voraussetzen muß, spricht umso mehr gegen ihn. Rationalität ist ein so allgemeiner Geltungsanspruch, daß er nicht mehr durch übergeordnete Kriterien bestätigt oder widerlegt werden kann - welche Kriterien immer man nennen würde, sie müssen ihrerseits bereits rationale sein. Dennoch kann eine indirekte Form

18 Stemmer (2000) 302-307.

19 Stemmer hebt das in einer Hinsicht deutlich hervor: „Man kann Wollenszustände ... nicht über ihre Inhalte kritisieren.“ $(2000,302)$ Aus dieser, wie ich meine, falschen Aussage zieht er andererseits nicht die Konsequenz, daß sich der Instrumentalist über die moralische Qualität der Motive gar nicht äussern sollte. In zugespitzter Form ist es für den Instrumentalisten ein Widerspruch, ein Buch über seine ethische Position zu veröffentlichen. Was will er damit erreichen? Meines Erachtens ist für eine solche öffentliche Handlungsweise eine Unterstellung nötig, die in der Argumentation Stemmers gar nicht verfügbar ist. 
des Bestätigens oder Kritisierens möglich sein. Indirekt bestätigt oder widerlegt sich eine Rationalitätsauffassung anhand der Folgen, die eintreten, wenn sie befolgt wird.

Eine bestimmte Auffassung von Rationalität muß, um selbst akzeptabel zu sein, selbstbestätigend sein. Selbstbestätigend ist eine Rationalitätsauffassung, wenn ihre Anwendung unter durchschnittlichen menschlichen Lebensumständen gute Folgen nach sich zieht, selbstdestruktiv ist sie, wenn sie schlechte Folgen nach sich zieht. Gute und schlechte Folgen müssen dabei unter dem Begriff der jeweiligen Rationalität selbst verstanden werden und nicht begriffsunabhängig. Dennoch ist das Kriterium nicht schlicht zirkulär, weil es eine offene empirische Frage ist, ob eine Rationalitätsauffassung sozial realisierbar ist oder nicht. Die Vermutung ist naheliegend, daß die instrumentelle Auffassung selbstdestruktiv ist, weil sie einen Beratungsbedarf weckt, aber nicht zu befriedigen vermag. Allgemein angewandt muß sie die sozialen Verhältnisse destabilisieren. ${ }^{20}$

In einer anderen Weise lässt sich dieses Kriterium auch folgendermaßen anwenden. Es ist hochgradig unplausibel, zu glauben, daß die von uns intuitiv als positiv angesehenen Qualitäten der sozialen Welt alle unabhängig von Rationalitätsansprüchen bestehen, gleichsam zufällig. Das müsste allerdings der Fall sein, wenn sie aus Interessen heraus entstünden, die ihrerseits nur empirisch kontingent sind. Im Gegensatz dazu liegt doch die Annahme viel näher, daß diesen positiven Qualitäten eine ,systematisierende Kraft‘ zugrundeliegt, die sich in einem zum instrumentellen alternativen Vernunftverständnis auch formulieren läßt. Von diesem Verständnis müsste man annehmen können, daß es nicht selbstdestruktiv, sondern selbstbestätigend ist: diejenigen, deren Handeln ihm entspricht, bestärken es und stabilisieren die von ihm beherrschten sozialen Beziehungen. Wie könnte ein solches Verständnis inhaltlich aussehen?

Einen Hinweis auf eine mögliche Antwort hat uns Kant gegeben. Kant hat nämlich deutlicher als jeder andere vor und nach ihm die Ansicht vertreten, daß Vernunft und Moral, und damit Vernunft und soziale Beziehungen intern zusammenhängen. Wenn er auch selbst in seiner praktischen Philosophie am Phänomen der Selbstbestätigung der Vernunft nicht besonders interessiert war, so ist doch klar, daß ein sozial selbstbestätigungsfähiger Vernunftbegriff notwendig ist für die soziale Stabilität zumindest einer aufklärten Moral. Andernfalls müsste man eine stabile Moral nur als eine unaufgeklärte, tradi-

20 Vielleicht wären sie tatsächlich „solitary, poore, nasty, brutish, and short“. - Die Idee einer Bewertung von praktischer Rationalität anhand ihrer Anwendung ist vor allem von Parfit (1984) Teil I, verfolgt worden. Parfit ist allerdings mehr daran interessiert, ob die Selbstinteresse-Konzeption selbstwiderlegend, nicht ob sie selbstbestätigend ist. 
tionelle Moral denken. Und in dieser Hinsicht scheint ein nicht-instrumenteller Begriff vorteilhafter. Während der instrumentelle die Willkür von Interessenvoraussetzungen zwar sichtbar werden läßt, sie aber nicht beheben kann, wird der nicht-instrumentelle Begriff nur die Willkür der faktischen Interessen beleuchten, während er die Notwendigkeit des Strebens und Handelns aus Gründen verdeutlicht.

Ein nicht-instrumentelles Vernunftverständnis muß eine ganze Reihe von Bedingungen erfüllen, deren Zusammenhang nicht auf Anhieb sichtbar ist. So muß es ebenfalls die Sein-Sollens-Problematik beantworten, muß zeigen, wie es zugleich empirisch und notwendig sein kann, und muß zusätzlich mit den eben genannten Bedingungen, vernünftig und moralisch, individuell und sozial zu sein, vereinbar sein. Dies ist nicht der Ort, um ein entsprechend komplexes Verständnis im Detail zu entwickeln. Dennoch will ich kurz darauf hinweisen, daß ein Vernunftverständnis selbstbestätigend sein könnte, wenn es nicht vom Begriff der Interessen, sondern von dem der Fähigkeiten ausgeht. Mithilfe einer Fähigkeitenkonzeption der Vernunft lassen sich auch die genannten Bedingungen am ehesten erfüllen, ohne daß dies jetzt im Detail geschildert werden könnte.

Klar scheint erstens, daß die Vernunftfähigkeit den allgemeinen begrifflichen Rahmen dafür liefern muß, was konkret vernünftig ist. Diese Fähigkeit konkretisiert sich in Gründen, und damit, entsprechend den früheren Bemerkungen, im Entstehen von Werten über das Anführen von Handlungszielen als Gründe für Handlungen. Während der Instrumentalist letztlich, weil er sich des Was seiner Wünsche als Gründe völlig enthalten will, bei willkürlichen und sinnlosen Gegebenheiten endet, gewinnt die nicht-instrumentelle Konzeption anhand des Was der Wünsche, oder eben der Gründe für bestimmte Wünsche, inhaltliche Werte. Wertvoll ist nach dieser Auffassung, was als wertvoll behauptet und vorläufig nicht widerlegt werden kann.

Im Unterschied zur Kantischen Vernunftlehre muß sich dieses Hervorbringen von Werten durch Gründe nicht unbedingt auf Metagründe oder allgemeinste Reflexionen über das menschliche Handeln beschränken. Das heißt, daß es nicht nur ,formale‘ Gründe, sondern auch ,materiale‘ Gründe sein können, Gründe im Rahmen der Anwendung vielfältiger menschlicher Fähigkeiten. Die menschliche Vernunft ist nicht nur eine formale, sondern eine materiale, eine, die in allen menschlichen Fähigkeiten wirksam und präsent ist. Nur so ist verständlich, daß auch solche Fähigkeiten wie Beobachten, Hören, Pflegen, Hämmern, Malen, Tennisspielen, usw. über das, was sie anzielen und woran sie ihrerseits zu messen sind, mehr oder weniger Wertvolles herstellen. Aus diesen fähigkeiteninvolvierten Gründen heraus wird klar, was an diesen und für diese Fähigkeiten gut ist.

Anhand von zwei einfachen Folgerungen wird nun leicht sichtbar, warum diese nicht-instrumentelle Vernunft selbstbestätigend wirken kann. Erstens und grundsätzlich: wenn die vernunftgeleitete Anwendung von Fähigkeiten 
Werte erzeugt, dann lässt die vermehrte Anwendung nur mehr Werte, also mehr Gutes als Schlechtes, entstehen. Die Grundidee ist einfach: Vernunft ist eine das Leben notwendig verbessernde und nicht verschlechternde Eigenschaft. Sie anzuwenden kommt allen zugute, die sie anwenden. Es kann nicht schlecht sein, sie anzuwenden, weil einzig ihre Anwendung die Werthaftigkeit des menschlichen Tuns erzeugt.

Zweitens, dieses Vernunftverständnis ist vor allem auch sozial selbstbestätigend, weil es diejenigen bestätigt, die im Besitz der Fähigkeiten sind, also die durchschnittlichen Menschen. Es wäre widersprüchlich, den Besitz der Fähigkeit für wertlos anzusehen, wenn die Fähigkeit es ist, aus der Wert entspringt. Und die menschlichen Personen sind in gewisser Weise der Besitz oder der Ort der Fähigkeiten. ${ }^{21}$ Die Personen werden, wenn sie ihre wichtigsten Fähigkeiten anwenden, damit auch erkennen, daß sie selbst wertvoll sind kraft ihrer Fähigkeiten, und das wird dazu führen, daß sie sich selbst und sich gegeneinander als gleichrangig erkennen, oder anders, einander achten dafür, daß sie die gleichen Fähigkeiten besitzen. Die Anwendung der Vernunftfähigkeit führt also dazu, universale Achtensbeziehungen zu verstärken, nicht sie zu untergraben. ${ }^{22}$

Alternativ ist die instrumentelle Konzeption nicht in der Lage, soziale Beziehungen des Achtens begrifflich auch nur zuzulassen, geschweige hervorzubringen. Der Begriff des Achtens ist ohne die Existenz von Werten oder Werthaftem nicht denkbar. Auch ein Gefühl oder ein Interesse des Achtens, wollte man sich darauf beziehen, ist als solches nicht formulierbar, wenn man nicht über den Begriff von Werten verfügt. Die globale Polemik des Instrumentalisten gegen Werte als ,metaphysische Fiktionen' hat die praktisch nicht ganz belanglose Konsequenz, daß eine soziale Welt sich achtender Personen als ebenso fiktiv gelten muß, wie die vermeintlich absonderlichen Werte. In der kontraktualistischen Gesellschaft haben die Beteiligten bestenfalls Interesse aneinander, sie können sich hingegen nicht gegenseitig achten. Es ist zweifelhaft, ob es den den Bewohnern dieser Gesellschaft erleichtern würde, dieses Defizit zu akzeptieren, wenn sie erkennen, daß sie ja auch keinen Grund haben, sich selbst zu achten.

21 Hier liegen offensichtlich einige begriffliche Fußangeln. Muß die Person nicht unabhängig davon bestehen, um Fähigkeiten zu haben? Bei personalitätsverleihenden Fähigkeiten kann allerdings das Verhältnis nicht äußerlich sein, ohne diese Fähigkeiten existiert keine Person.

22 Vorausgesetzt ist dabei, daß der unterschiedliche Besitz von konkreten Fähigkeiten nicht mit entsprechenden Werthierarchien der Fähigkeiten und dann der Personen einhergeht. Ein Grund, warum das naheliegt, ist die herausragende Rolle der Vernunft auch für alle materialen Fähigkeiten. Für Beobachtungen zu diesem und anderen Punkten s. auch Raz (2001) Kap. 4. Raz lässt allerdings im unklaren, wie er den Wertbegriff einführen will. 


\section{6. Öffentliche Moral und Kontraktualismus}

Alle Moralphilosophen müssen, wenn sie ihre Argumente ernst nehmen, diese letztlich auch der Öffentlichkeit gegenüber vertreten können - sie also der Öffentlichkeit anbieten. Insgesamt heißt das, daß die philosophisch zu rechtfertigende Moral, oder Teile aus ihr, auch die Form einer öffentlichen Moral annehmen können muß. Was ist mit dieser Anforderung an die Moral gemeint? Öffentlich ist eine Moral dann, wenn in ihr ein Bewußtsein davon herrscht, daß Teile der Moral, und genauer für das Zusammenleben unverzichtbare Teile, durch ein gemeinsames Beraten erst gefunden werden müssen. In Anschluß an die bisherigen Bemerkungen zur Selbstbestätigung wirft der Begriff der öffentlichen Moral eine speziellere Frage der politischen Realisierbarkeit der Moral auf. Generell werden soziale Realisationsfragen zur Moral in der philosophischen Ethik gern vernachlässigt. ${ }^{23} \mathrm{Da}$ die politischen Institutionen moralische Grundlagen haben und fortwährend auch in moralischer Hinsicht weiter entwickelt werden müssen, sollte jede philosophische Begründung der Moral jedoch diese spezielle Anforderung im Blick haben: inwieweit sie eine politische Beratung über die moralischen Grundlagen der Politik ermöglicht.

Es sind sicher im Detail verschiedene Konzeptionen eines politisch-moralischen Diskurses möglich. Sie alle müssen jedoch die Eigenschaft haben, daß die Beteiligten einander in bestimmtem Ausmaß bereits moralische Rechte zuerkennen, um unter diesen Bedingungen über weitere Rechte, oder die Details von Rechten, zu diskutieren. Es könnte nun so aussehen, daß der Begriff einer öffentlichen Moral, wenn man in ihm nur moralische Grundrechte einbezieht, bereits alle Anforderungen erfüllt, die an einen solchen Begriff zu stellen sind. Wird dann nicht eine öffentliche Diskussion über unklare und offene moralische Fragen möglich, auf der Basis eben der garantierten und selbst nicht offenen moralischen Rechte? Genauer stellt sich aber die Frage, ob eine solche Zweiteilung der öffentlichen Moral mit beliebigen Begründungen der von ihr als garantiert vorausgesetzten moralischen Rechte vereinbar ist. Eine kontraktualistische Begründung erfüllt meines Erachtens diese Anforderung einer öffentlichen Moral nicht.

Ein erster Hinweis zugunsten dieser Vermutung liegt in dem Eingeständnis, daß die kontraktualistische Moral keine universelle und egalitäre sein kann. ${ }^{24}$ Es ist kaum anzunehmen, daß eine öffentliche Moralkultur als solche

23 Eine berühmte Ausnahme ist H. Sidgwick. Sidgwick war wesentlich skeptischer gegenüber der aufklärerisch-positiven Wirkung des Utilitarismus als es die klassischen Utilitaristen Bentham, Hume oder Mill waren, und er sah deutlicher die mögliche Gefahr der sozial-politischen Selbstdestruktivität gerade des Utilitarismus. S. Sidgwick (1901) ch. IV.iii.

24 Stemmer (2000) $\$ \mathbb{S} 7-8$ 
sozial funktioniert, wenn in ihr auch fortwährend darüber diskutiert werden muß, ob Behinderte, Alte, Kinder und generell Menschen mit unterschiedlichem sozialen Status dieselben Rechte haben können wie die übrigen. Die öffentliche Diskussion über solche Differenzen wird dadurch erschwert, daß die Beiträge zu ihr vonseiten der Beteiligten ihrerseits von deren sozialem Status abhängen, also davon, ob sie selbst mehr oder weniger droh- oder kooperationsfähig sind. Damit wird ein gegenseitiges Beraten oder Deliberieren unmöglich, weil Argumente ersetzt werden durch Motive, diskursive Rationalität durch motivationale Rationalität. Das Argument des Starken gegenüber dem Schwachen reduziert sich darauf, daß er stark ist - und das ist ohne ein entsprechendes Prinzip kein Argument. Ich gehe zunächst einmal davon aus, daß ein geeignetes Prinzip im kontraktualistischen Gedankengang nicht zur Verfügung steht.

$\mathrm{Da}$ die Verteidigung verschiedener Standpunkte in einer kontraktualistischen Moral, wie Stemmer sie vorstellt, aus realen Machtunterschieden heraus vorgetragen werden muß, liegt wiederum am instrumentellen Rationalitätsbegriff. Nach diesem sind die praktischen Gründe durch die faktischen Interessen bedingt, und in den reziproken sozialen Beziehungen bedeuten die Schwächen des einen ein geringeres Interesse des anderen, mit ihm zu kooperieren, also ihm dieselben Rechte einzuräumen wie denjenigen, von denen seine Interessen stärker bedient werden. Reale Differenzen in der Handlungsfähigkeit übersetzen sich unmittelbar in normative Hierarchien des moralischen Status und von moralischen Rechten.

Stemmer ist sich dieses Einwands, der im übrigen jeden Unterschied zwischen dem tatsächlichen Wünschen und Handeln, den tatsächlichen sozialen Verhältnissen und einem moralischen Ideal dieser Verhältnisse beseitigen würde, durchaus bewußt. In einer Antwort auf ihn versucht er zu unterscheiden zwischen einem erpresserischen Müssen, das durch einen Räuber veranschaulicht wird, und dem moralischen Müssen, das sich neben der reinen Klugheit, die auch für das erpresserische Müssen gilt, außerdem einem autonomem Zugeständnis verdankt. ${ }^{25} \mathrm{Daß}$ ein solcher Rückgriff auf Autonomie für Stemmer überhaupt verfügbar ist, scheint mir jedoch zweifelhaft.

Kein Unterschied besteht offensichtlich zwischen dem Handeln aus Klugheit gegenüber dem Räuber und einem klugen Kooperieren in der kontraktualistischen Moral, wenn man eine schwächere Position innehat. In beiden Fällen handelt man aus Klugheit, und in beiden Fällen handelt man unter externem Zwang. Daß man einverständig handelt, also kooperiert, ist kein Beleg dafür, daß man autonom handelt. Die Autonomie wird eingeschränkt

25 Ebd. 110; auch Stemmer in diesem Band, 42, 57, 62, bes. 64. 
durch den externen Zwang. Inhaltlich andere, aber strukturell dieselben Verhältnisse bestehen unter denen, die aus einer gleichen Position der Stärke kooperieren, was nach Annahme der meisten Kontraktualisten für die Mehrzahl der Gesellschaftsmitglieder gilt. Das Einverständnis, zu kooperieren, wird erleichtert durch das Wissen, daß die anderen in derselben Lage sind: der selbst auferlegte Zwang wird erleichtert durch das Wissen, daß die anderen sich denselben Zwang auferlegen. Das Wissen, daß gleiche Verhältnisse herrschen, ändert aber nichts daran, daß nur aus Klugheit und unter Zwang gehandelt wird. Externen Zwang enthalten auch die egalitären Verhältnisse, weil die Existenz der anderen zwingt, sich zu beschränken.

Im Ergebnis sollte Stemmer den Unterschied nicht mithilfe von Autonomie beantworten, sondern mit einem Verweis auf die egalitären Verhältnisse. Allerdings bleibt dann unklar, in welchem Ausmaß solche Verhältnisse bestehen. Im Unterschied zu unserem üblichen Ethos der universalen Menschenrechte, das Gleichheit prinzipiell allen normativ zugesteht, bliebe es in der realistisch gefaßten kontraktualistischen Moral immer von den tatsächlichen Interessen und Fähigkeiten abhängig, in welchem Umfang und unter genau welchen Mitgliedern egalitäre Verhältnisse herrschen. Moralische Gleichheit stünde immer unter einem Vorbehalt, die Beteiligten am (gewissermaßen) ,Gleichheitsbereich` der Moral müssten ihre Berechtigung, in diesen Bereich zu fallen, fortwährend ausweisen. Im Grunde wäre ein fortwährendes Aushandeln, oder ein Begutachten durch eine Behörde, erforderlich, um die sozialen Beziehungen ,moralisch`zu regeln.

Im Licht dieser Konsequenzen wird sichtbar, daß die Hobbessche und die Humesche Version des Kontraktualismus nicht einfach nur ,Betriebsunfälle' dieses Programms waren, die man durch Rücknahme einiger Prämissen leicht vermeiden könnte. In der Hobbesschen Theorie wird Moral in Politik, genauer in der staatlichen Macht, aufgelöst. Eine stärkere und neben der Politik bestehende Sozialmoral wäre nach Hobbes nur hinderlich, und natürlich ist ein etablierter öffentlicher moralischer Diskurs mit seiner Politikauffassung unvereinbar. Dasselbe gilt aber auch für Hume. In der Humeschen Theorie wird Moral in den Gewohnheiten und Konventionen aufgelöst. Eine verbreitete Diskussion über Gerechtigkeit gilt ebenfalls als unerwünscht. ${ }^{26}$ Eine Erklärung dieser Furcht vor öffentlicher Diskussion ist jetzt naheliegend: Für das Ermitteln der Moral stehen keine anderen Gründe zur Verfügung als die sozialen Verhältnisse selbst, und das öffentliche Normieren dieser Verhältnisse würde mehr soziale Konflikte hervorbringen als beseitigen. Zumindest in einer aufgeklärten Gesellschaft ist die kontraktualistische Moral also schwer denkbar.

26 S. die Kritik der Leveller in I, Sect. III, part II. 
Indem die meisten Kontraktualisten ein rein bypothetisches Begründungsverständnis haben, sehen sie vermutlich ihr Begründen von einem solchen öffentlichen Realisierungsanspruch gar nicht bedroht. Das wäre jedoch ein Mißverständnis, weil jede Moralbegründung eine öffentlich-praktische Bedeutung haben sollte, und zwar mindestens in der Theorie. ${ }^{27}$ Selbst wenn solche Begründungen in der realen Welt wenig wahrgenommen werden, scheint es widersinnig, daß sie gar nicht wahrgenommen werden dürfen. Eine Moral, deren Praxis fatale Folgen hätte, kann keine akzeptable Moral sein. Davon abgesehen besteht in demokratischen Gesellschaften ja ein gewisses Ausmaß an tatsächlicher öffentlicher moralischer Diskussion. Die Grundlagen der Moral sind aus dieser Diskussion, wie etwa philosophische Beiträge zur Ethik des frühen menschlichen Lebens zeigen, nicht auszugrenzen. Die Grundlagen werden damit schnell in den öffentlichen Diskurs einbezogen und entwickeln dann die hier geschilderte Wirkung.

Es ist also nicht zu sehen, wie der Kontraktualismus eine öffentliche Moral ermöglichen soll. Eine öffentliche Moral setzt Grundrechte voraus, deren Anerkennung vom tatsächlichen sozialen Status, dem Innehaben von mehr oder weniger sozialer Macht, frei ist und die vom Erlebnis der partiellen Offenheit der Moral nicht erodiert werden können. Nur eine Vernunftbegründung der Moral wird eine dynamisch weiter zu entwickelnde öffentliche Moral ermöglichen, die selbst von kontingenten sozialen Verhältnissen frei ist. Die instrumentelle praktische Vernunft ist in dieser Hinsicht ungeeignet, eben weil sie notwendig von den tatsächlichen sozialen Verhältnissen abhängig bleibt. Demgegenüber ist die einzig auf Gründen beruhende nicht-instrumentelle Vernunft nicht nur kontingenzfrei, sie widersteht auch der Skepsis, wie sie in der öffentlichen Moral notgedrungen aufkommt.

\section{Ein Vorschlag}

Im Kern resultiert die Unfähigkeit des Kontraktualismus zu einer öffentlichen Moral aus seiner Reduktion von Gründen auf Motive und Interessen, oder (anders gesagt) von Gründen auf soziale Fakten und Verhältnisse. Eine sozial stabile Form der Gleichheit muß durch Gründe erzengt werden, und der instrumentelle Begriff der Gründe ist dazu ungeeignet. Umgekehrt heißt das, daß eine sozial stabile öffentliche Moral ein Gleichheitsprinzip voraussetzt,

27 Jede Ethik sollte mindestens eine praktische Bedeutung haben, also in ihrer Rechtfertigung dem konkreten Handeln nach ihren Prinzipien Rechnung tragen. Bei Theorien, die explizit kollektiv-begründende Argumente vorbringen, muß die praktische Bedeutung eben deshalb eine öffentlich-praktische Bedeutung sein. Der Kontraktualismus gehört in diese Kategorie. 
das nicht auf unterschiedliche soziale Verhältnisse, tatsächliche Machtunterschiede, reduzierbar ist. Es ist offensichtlich, daß der auf dem instrumentellen Begriff beruhende Kontraktualismus ein solches Prinzip explizit nicht zur Verfügung stellen kann.

Andererseits könnte man ihn allerdings so interpretieren, daß er es implizit voraussetzt. Ein Begründungsvorschlag, wie ihn etwa Stemmer artikuliert, ist ja als ein Angebot an alle gemeint - alle werden eingeladen, sich in die Rolle des Skeptikers zu begeben. Damit wird ein Schritt in die öffentliche Moral getan, der zwar nicht begründet ist, aber als solcher unterstellt werden sollte. Der Kontraktualismus kann nur so verstanden werden: als ein Vorschlag, Prinzipien der Gerechtigkeit im Rahmen einer bereits vorausgesetzten Gleichheit zu ermitteln. Macht wird dann ersetzt durch Verdienst, und verdienstliche Differenzen haben ihren berechtigten Ort.

\section{Literatur}

Lev: T. Hobbes, Leviathan, London 1651.

EPM: D. Hume, An Enquiry Concerning the Principles of Morals, Edinburgh 1751.

Brandt, R. (1979), A Theory of the Good and the Right, Oxford.

Mackie, J. L. (1977), Ethics. Inventing Right and Wrong, Harmondsworth. Parfit, D. (1984), Reasons and Persons, Oxford.

Raz, J. (2001), Value, Respect, and Attachment, Cambridge.

Scanlon, T. (1998), What We Owe to Each Other, Cambridge, Mass.

Sidgwick, H. (1901), The Methods of Ethics, 6. Aufl. London.

Stemmer, P. (2000), Handeln zugunsten anderer, Berlin/New York. 